Document downloaded from:

http://hdl.handle.net/10251/47377

This paper must be cited as:

Denia, FD.; Martínez-Casas, J.; Baeza, L.; Fuenmayor, FJ. (2012). Acoustic modelling of exhaust devices with nonconforming finite element meshes and transfer matrices. Applied Acoustics. 73(8):713-722. doi:10.1016/j.apacoust.2012.02.003.

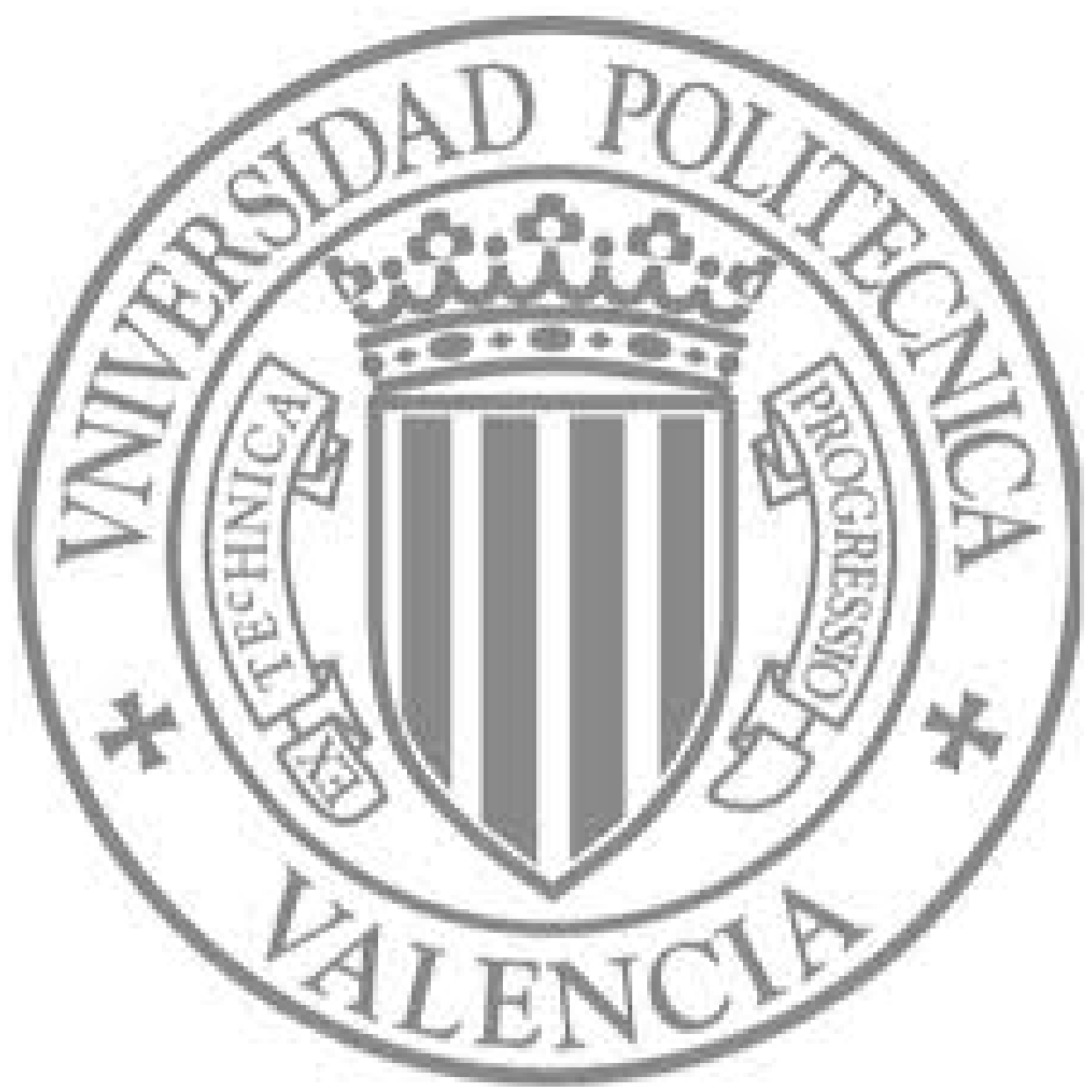

The final publication is available at

http://dx.doi.org/10.1016/j.apacoust.2012.02.003

Copyright Elsevier 


\title{
Acoustic modelling of exhaust devices with nonconforming finite element meshes and transfer matrices
}

\author{
F. D. Denia , J. Martínez-Casas, L. Baeza, F. J. Fuenmayor \\ Centro de Investigación de Tecnología de Vehículos, Universitat Politècnica de \\ València, Camino de Vera s/n, 46022 Valencia, Spain
}

14 November 2011

Keywords: Nonconforming meshes, finite elements, transfer matrices

* Corresponding author:

Dr. F. D. Denia

Centro de Investigación de Tecnología de Vehículos

Universitat Politècnica de València

Camino de Vera $\mathrm{s} / \mathrm{n}$

46022 Valencia

Spain

Tl: + 34963879620

Fax: + 34963877629

e-mail: fdenia@mcm.upv.es 


\section{ABSTRACT}

Transfer matrices are commonly considered in the numerical modelling of the acoustic behaviour associated with exhaust devices in the breathing system of internal combustion engines, such as catalytic converters, particulate filters, perforated mufflers and charge air coolers. In a multidimensional finite element approach, a transfer matrix provides a relationship between the acoustic fields of the nodes located at both sides of a particular region. This approach can be useful, for example, when one-dimensional propagation takes place within the region substituted by the transfer matrix. As shown in recent investigations, the sound attenuation of catalytic converters can be properly predicted if the monolith is replaced by a plane wave four-pole matrix. The finite element discretization is retained for the inlet/outlet and tapered ducts, where multidimensional acoustic fields can exist. In this case, only plane waves are present within the capillary ducts, and three-dimensional propagation is possible in the rest of the catalyst subcomponents. Also, in the acoustic modelling of perforated mufflers using the finite element method, the central passage can be replaced by a transfer matrix relating the pressure difference between both sides of the perforated surface with the acoustic velocity through the perforations. The approaches in the literature that accommodate transfer matrices and finite element models consider conforming meshes at connecting interfaces, therefore leading to a straightforward evaluation of the coupling integrals. With a view to gaining flexibility during the mesh generation process, it is worth developing a more general procedure. This has to be valid for the connection of acoustic subdomains by transfer matrices when the discretizations are nonconforming at the connecting interfaces. In this work, an integration algorithm similar to those considered in the mortar finite element method, is implemented for nonmatching grids in combination with 
acoustic transfer matrices. A number of numerical test problems related to some relevant exhaust devices are then presented to assess the accuracy and convergence performance of the proposed procedure. 


\section{INTRODUCTION}

The use of transfer matrices [1] is a widespread practice in the acoustic modelling of ducts and mufflers. This approach is also applied to additional devices found in the breathing system of internal combustion engines, which have an impact on the control of acoustic emissions as well: catalytic converters [2-4], particulate filters [4,5] and charge air coolers [6]. Transfer matrices can be incorporated into multidimensional modelling tools based on the finite element (FE) method and the boundary element (BE) method [7-9] to predict the acoustic behaviour of these devices.

The application of FE/BE approaches to catalytic converters has been presented in a number of investigations [2,10-12]. Two alternative modelling techniques are available for the monolith. The first model consists of assuming equivalent acoustic properties, similar to a homogeneous and isotropic bulk-reacting absorbent material [2,13]. In this case, the numerical approach computes three-dimensional acoustic fields inside all the catalytic converter components, including the inlet/outlet ducts and the monolith [2]. The second model replaces the monolith by a plane wave connection or a "element-to-element four-pole transfer matrix" [10-12]. This approach provides a relationship between the acoustic fields associated with the discretizations located at both sides of the monolithic region. The acoustic behaviour of the capillary ducts is one-dimensional, while three-dimensional acoustic waves can still be present in the inlet/outlet ducts. Although this second approach seems more consistent with the actual acoustic phenomena inside the capillaries, the predictions of both techniques can exhibit a reasonable agreement in comparison with the experimental measurements, depending on the particular characteristics of the configuration under analysis. Attention has also been paid to the numerical modelling of particulate filters $[4,5,11]$. The 
combination of a multidimensional BE simulation with transfer matrices was presented in Ref. [11]. An acceptable agreement between predictions and measurements was found.

Concerning the acoustic modelling of mufflers with perforated pipes, numerous works are now available in the bibliography [14-21]. A number of these references [17-21] include multidimensional analytical and/or numerical models for dissipative configurations with absorbent material. Additional considerations can be found in Refs. [20,21] related to the presence of mean flow in the perforated central passage. In all the cases, numerical results from $\mathrm{FE} / \mathrm{BE}$ calculations are presented, as a main contribution of the work or as a reference solution to validate an analytical approach. The perforated surface is usually modelled by its acoustic impedance, which relates the pressure and velocity at both sides of the perforations. These sides are discretized into two identical overlapped meshes with coincident nodes. From a numerical point of view, the introduction of the perforated screen in a numerical technique such as the FE method can be considered as a particular situation of the general transfer matrix approach, as will be detailed later in Sec. 3.2. In this case the diagonal terms of the four-pole transfer matrix [1] are equal to unity, the off-diagonal term $(2,1)$ is zero and the offdiagonal term $(1,2)$ equals the acoustic impedance of the perforated surface.

Despite extensive literature devoted to FE/BE models for mufflers, catalysts and filters, a common feature is the use of conforming discretizations at the boundaries coupled through the transfer matrix. In all the cases, the meshes of the connected subdomains match on the interface. The numerical computations are simplified but the flexibility of the mesh generation process is reduced. For example, in the FE modelling of complex mufflers with perforated ducts [16] the discretization technique is time consuming and tedious, since two identical overlapped grids with duplicated nodes must be generated at the interfaces of each 
perforated screen. Similar comments can be applied to the discretization associated with both sides of a catalytic converter [12]. The need of conforming meshes at the boundary interfaces coupled by the transfer matrix requires the use of special meshing operations, depending on the particular geometry under analysis. These operations may include mesh reflection (if both sides are symmetric) or 2D mesh translation from one side to the other, followed by $3 \mathrm{D}$ mesh generation from a 2D base grid. Therefore, mesh generation can be computationally expensive compared to situations where conformity is not necessary. In addition, these cumbersome algorithms are not always valid, since the connecting interfaces at both sides of the monolith can have different geometries in same cases, thus requiring nonconforming discretizations. The latter have received attention during the last two decades, particularly in problems that concern solid and contact mechanics [22-24]. Regarding the numerical modelling of acoustic and vibroacoustic problems, some reported attempts have been found in the literature related to nonconforming meshes [25-27], with a view to taking advantage of more flexible discretization techniques. In these works, the authors considered nonmatching discretizations in coupled mechanical-acoustic systems and also acoustic-acoustic coupling problems, without including the presence of a transfer matrix. In the vibroacoustic problem, the elements associated with the mesh within the solid are usually smaller than the elements of the fluid discretization. Different physical fields (displacements in the solid and velocity potential or acoustic pressure within the fluid) are coupled over nonconforming interfaces where the nodes do not coincide, taking into account proper continuity conditions. Therefore, the mesh creation for a subdomain does not require information from other subdomains. In the acoustic-acoustic problem, the same physical field (velocity potential or acoustic pressure) is coupled by Lagrange multipliers over a nonconforming interface. Applications are related to flow induced noise calculations [27], where the interface separates two regions: the aeroacoustic subdomain, with a smaller element size, associated with the fluid flow problem 
(and therefore the source terms), and the purely acoustic subdomain, where the homogeneous wave equation is solved. Since the FE mesh is nonconforming at the interface, the continuity of acoustic pressure is not fulfilled directly, and must be enforced in a weak sense with suitable Lagrange multipliers [23,27] In some cases [25], this procedure exhibits better computational behaviour than the conforming FE version, where a small transition region from fine to coarse mesh is considered.

In Refs. [25] and [27], a direct contact exists between the different propagation media. Therefore, continuity conditions of the relevant physical fields are used in the formulation (for example, continuity of velocity and pressure in the acoustic-acoustic coupling problem). In the current investigation, the propagation media are separated by a connecting region, and there is no direct contact between them. From a practical point of view, this situation is quite common in devices such as perforated mufflers and catalytic converters, where pressure and velocity changes can occur through the connecting region. This region is replaced by a transfer matrix and discontinuous fields, such as acoustic pressure and velocity, are permitted in the acoustic-acoustic coupling over nonmatching interfaces.

The main goal of the current investigation is to examine the numerical performance of the nonconforming version of the FE method for modelling acoustic systems with subdomains coupled by means of transfer matrices. Here, the continuity conditions of the acoustic fields at the interfaces [25-27] are replaced by four-pole relationships between the acoustic pressure and velocity at both sides of the subsystem represented through a transfer matrix. Applications of practical interest are related to a number of devices used in the exhaust system of internal combustion engines, such as perforated ducts, catalytic converters and particulate filters. Following this Introduction, this work begins by revising the FE equations 
for two subdomains coupled by a transfer matrix (Sec. 2). Details are also presented concerning the integration procedure to evaluate the coupling integrals in nonconforming meshes. Sec. 3 provides the main details of the transfer matrices for the numerical test problems, consisting of a catalytic converter and a perforated dissipative muffler. To focus on the convergence behaviour of the nonconforming approach, the geometries of the particular configurations under consideration are relatively simple. For these two exhaust devices, this section presents the FE results with conforming and nonmatching meshes. A comparison is carried out considering the accuracy and convergence performance, for some relevant acoustic magnitudes, such as the four poles. The work concludes in Sec. 4 with some final remarks.

\section{NUMERICAL APPROACH}

\subsection{Finite element equations}

Fig. 1(a) shows the sketch of an acoustic device, which consists of three subdomains denoted by $\Omega_{1}, \Omega_{c}$ and $\Omega_{2}$. In addition, $\Gamma_{1 b c}$ and $\Gamma_{2 b c}$ denote the contour of subdomains $\Omega_{1}$ and $\Omega_{2}$ respectively, where Neumann boundary conditions are applied, while $\Gamma_{1 c}$ and $\Gamma_{2 c}$ represent the coupling interfaces $\Omega_{1} / \Omega_{c}$ and $\Omega_{2} / \Omega_{c}$. Fig. 1(b) depicts the associated finite element mesh, nonconforming at the interfaces $\Gamma_{1 c}$ and $\Gamma_{2 c}$. As can be seen, the connecting subdomain $\Omega_{c}$ has been replaced by a transfer matrix $\mathbf{T}$ [10-12], thus establishing a relation between the acoustics fields within $\Omega_{1}$ and $\Omega_{2}$. The propagation medium is assumed homogeneous and isotropic, characterized by the densities $\rho_{1}$ and $\rho_{2}$, and speeds of sound $c_{1}$ and $c_{2}$ for the subdomains $\Omega_{1}$ and $\Omega_{2}$, respectively. 
The sound propagation is governed by the well-known Helmholtz equation [1]

$$
\nabla^{2} P_{i}+k_{i}^{2} P_{i}=0, \quad i=1,2
$$

where $\nabla^{2}$ is the Laplacian operator, $P_{i}$ is the acoustic pressure within subdomain $\Omega_{i}$, and $k_{i}=\omega / c_{i}$ is the associated wavenumber, defined as the ratio of the angular frequency $\omega$ to the corresponding speed of sound.

To derive the finite element equations associated with Eq. (1), the method of weighted residuals can be used in combination with the Galerkin approach [23]. For the sake of clarity, the most relevant equations are detailed next. Using Gauss' theorem, Eq. (1) leads to

$$
\int_{\Omega_{i}} \nabla W_{i} \nabla P_{i} \mathrm{~d} \Omega-k_{i}^{2} \int_{\Omega_{i}} W_{i} P_{i} \mathrm{~d} \Omega=\int_{\Gamma_{i b c}} W_{i} \frac{\partial P_{i}}{\partial n} \mathrm{~d} \Gamma+\int_{\Gamma_{i c}} W_{i} \frac{\partial P_{i}}{\partial n} \mathrm{~d} \Gamma, \quad i=1,2,
$$

with $W_{i}$ being a weighting function and $n$ representing the outward normal to the boundary. The coupling between the interfaces $\Gamma_{1 c}$ and $\Gamma_{2 c}$ associated with both sides of the connecting subdomain $\Omega_{c}$ is carried out by using a transfer matrix $\mathbf{T}$ [10-12]. Details of the particular expressions for $\mathbf{T}$ considered in the current investigation will be provided in Sec. 3 for several test problems including a catalytic converter and a perforated dissipative muffler. Here, the usual four-pole matrix relating pressure and velocity upstream (subscript 1) with the same fields downstream (subscript 2) is considered [1],

$$
\left\{\begin{array}{c}
P_{1} \\
U_{1}
\end{array}\right\}=\mathbf{T}\left\{\begin{array}{c}
P_{2} \\
U_{2}
\end{array}\right\}=\left(\begin{array}{ll}
T_{11} & T_{12} \\
T_{21} & T_{22}
\end{array}\right)\left\{\begin{array}{c}
P_{2} \\
U_{2}
\end{array}\right\} .
$$

Using Euler's equation [1], the velocity and the normal derivative of the pressure are related. Therefore, the following relations are satisfied

$$
\begin{gathered}
P_{1}=T_{11} P_{2}-T_{12}\left(\frac{-1}{\mathrm{j} \omega \rho_{2}} \frac{\partial P_{2}}{\partial n}\right), \\
\frac{-1}{\mathrm{j} \omega \rho_{1}} \frac{\partial P_{1}}{\partial n}=T_{21} P_{2}-T_{22}\left(\frac{-1}{\mathrm{j} \omega \rho_{2}} \frac{\partial P_{2}}{\partial n}\right) .
\end{gathered}
$$


The sign changes for $T_{12}$ and $T_{22}$ in Eqs. (4) and (5) account for the sign of the normal velocities over the interfaces $\Gamma_{1 c}$ and $\Gamma_{2 c}$ chosen for the calculations ( $U_{1}$ points outward the subdomain $\Omega_{1}$, thus similar to $n$, and $U_{2}$ is directed normally inward $\Omega_{2}$, opposite to $n$ ). After manipulation of Eq. (4),

$$
\frac{\partial P_{2}}{\partial n}=\frac{\mathrm{j} \omega \rho_{2}}{T_{12}} P_{1}-\frac{\mathrm{j} \omega \rho_{2} T_{11}}{T_{12}} P_{2}=\mathrm{j} \omega \Pi_{21} P_{1}-\mathrm{j} \omega \Pi_{22} P_{2} .
$$

Combining Eqs. (5) and (6)

$$
\frac{\partial P_{1}}{\partial n}=-\mathrm{j} \omega \frac{\rho_{1}}{\rho_{2}} T_{22} \Pi_{21} P_{1}+\mathrm{j} \omega\left(\frac{\rho_{1}}{\rho_{2}} T_{22} \Pi_{22}-\rho_{1} T_{21}\right) P_{2}=-\mathrm{j} \omega \Pi_{11} P_{1}+\mathrm{j} \omega \Pi_{12} P_{2} .
$$

Now Eq. (7) is introduced in the second term (right-hand side) of the weighted residual expressed in Eq. (2), for $i=1$ (subdomain $\Omega_{1}$ ). For a suitable discretization, within a typical element it is assumed

$$
P_{i}(x, y, z)=\mathbf{N}_{i} \tilde{\mathbf{P}}_{i}, \quad i=1,2,
$$

with $\mathbf{N}_{i}$ containing the shape (or interpolation) functions of the nodes and $\tilde{\mathbf{P}}_{i}$ the nodal values. According to the Galerkin approach, the weighting functions are chosen to be the same as the shape functions. Incorporating Eq. (8) in Eq. (2), the weighted residual leads to the FE matrizant system of equations. After assembly, this system can be written in compact form as

$$
\left(\mathbf{K}_{1}+\mathrm{j} \omega \mathbf{C}_{1}-\omega^{2} \mathbf{M}_{1}\right) \tilde{\mathbf{P}}_{1}-\mathrm{j} \omega \mathbf{C}_{12} \tilde{\mathbf{P}}_{2}=\mathbf{F}_{1}
$$

In Eq. (9), the following nomenclature has been introduced

$$
\begin{gathered}
\mathbf{K}_{1}=\sum_{e=1}^{N_{1}^{e}} \int_{\Omega_{1}^{e}} \nabla^{\mathrm{T}} \mathbf{N}_{1} \nabla \mathbf{N}_{1} \mathrm{~d} \Omega, \\
\mathbf{C}_{1}=\Pi_{11} \sum_{e=1}^{N_{1 c}^{e}} \int_{\Gamma_{1 c}^{e}} \mathbf{N}_{1}^{\mathrm{T}} \mathbf{N}_{1} \mathrm{~d} \Gamma, \\
\mathbf{M}_{1}=\frac{1}{c_{1}^{2}} \sum_{e=1}^{N_{1}^{e}} \int_{\Omega_{1}^{e}} \mathbf{N}_{1}^{\mathrm{T}} \mathbf{N}_{1} \mathrm{~d} \Omega,
\end{gathered}
$$




$$
\begin{gathered}
\mathbf{C}_{12}=\prod_{12} \sum_{e=1}^{N_{1 c}^{e}} \int_{\Gamma_{1 c}^{e}} \mathbf{N}_{1}^{\mathrm{T}} \mathbf{N}_{2} \mathrm{~d} \Gamma, \\
\mathbf{F}_{1}=\sum_{e=1}^{N_{1 b c}^{e}} \int_{\Gamma_{1 b c}^{e}} \mathbf{N}_{1}^{\mathrm{T}} \frac{\partial P_{1}}{\partial n} \mathrm{~d} \Gamma,
\end{gathered}
$$

where $\Sigma$ denotes a finite element assembly operator, $N_{1}^{e}$ represents the number of domain elements in the discretization of the subdomain $\Omega_{1}, N_{1 b c}^{e}$ the number of contour elements associated with boundary conditions and $N_{1 c}^{e}$ the number of contour elements located on the coupling interface $\Gamma_{1 c}$.

Substituting now Eq. (6) in the second term of the weighted residual expressed in Eq. (2), for $i=2$ (subdomain $\Omega_{2}$ ), and applying the FE approach, yields

$$
\left(\mathbf{K}_{2}+\mathrm{j} \omega \mathbf{C}_{2}-\omega^{2} \mathbf{M}_{2}\right) \tilde{\mathbf{P}}_{2}-\mathrm{j} \omega \mathbf{C}_{21} \tilde{\mathbf{P}}_{1}=\mathbf{F}_{2},
$$

with the notation

$$
\begin{gathered}
\mathbf{K}_{2}=\sum_{e=1}^{N_{2}^{e}} \int_{\Omega_{2}^{e}} \nabla^{\mathrm{T}} \mathbf{N}_{2} \nabla \mathbf{N}_{2} \mathrm{~d} \Omega, \\
\mathbf{C}_{2}=\Pi_{22} \sum_{e=1}^{N_{2 c}^{e}} \int_{\Gamma_{2 c}^{e}} \mathbf{N}_{2}^{\mathrm{T}} \mathbf{N}_{2} \mathrm{~d} \Gamma, \\
\mathbf{M}_{2}=\frac{1}{c_{2}^{2}} \sum_{e=1}^{N_{2}^{e}} \int_{\Omega_{2}^{e}} \mathbf{N}_{2}{ }^{\mathrm{T}} \mathbf{N}_{2} \mathrm{~d} \Omega, \\
\mathbf{C}_{21}=\prod_{21} \sum_{e=1}^{N_{2 c}^{e}} \int_{\Gamma_{2 c}^{e}} \mathbf{N}_{2}^{\mathrm{T}} \mathbf{N}_{1} \mathrm{~d} \Gamma, \\
\mathbf{F}_{2}=\sum_{e=1}^{N_{2 b c}^{e}} \int_{\Gamma_{2 b c}^{e}} \mathbf{N}_{2}{ }^{\mathrm{T}} \frac{\partial P_{2}}{\partial n} \mathrm{~d} \Gamma .
\end{gathered}
$$


Eqs. (9) and (15) are written as

$$
\left(\left(\begin{array}{cc}
\mathbf{K}_{1} & \mathbf{0} \\
\mathbf{0} & \mathbf{K}_{2}
\end{array}\right)+\mathrm{j} \omega\left(\begin{array}{cc}
\mathbf{C}_{1} & -\mathbf{C}_{12} \\
-\mathbf{C}_{21} & \mathbf{C}_{2}
\end{array}\right)-\omega^{2}\left(\begin{array}{cc}
\mathbf{M}_{1} & \mathbf{0} \\
\mathbf{0} & \mathbf{M}_{2}
\end{array}\right)\right)\left\{\begin{array}{l}
\tilde{\mathbf{P}}_{1} \\
\tilde{\mathbf{P}}_{2}
\end{array}\right\}=\left\{\begin{array}{l}
\mathbf{F}_{1} \\
\mathbf{F}_{2}
\end{array}\right\},
$$

or, in compact form, as

$$
\left(\mathbf{K}+\mathrm{j} \omega \mathbf{C}-\omega^{2} \mathbf{M}\right) \tilde{\mathbf{P}}=\mathbf{F} .
$$

It is worth noting that the matrix $\mathbf{C}$ contains the acoustic information associated with the transfer matrix $\mathbf{T}$.

\subsection{Integration of coupling matrices over nonconforming meshes}

The evaluation of the coupling integrals involved in $\mathbf{C}_{12}$ and $\mathbf{C}_{21}$, whose detailed expressions are given in Eqs. (13) and (19), is relatively simple for conforming meshes, since in this case the shape functions are equal, $\mathbf{N}_{1}=\mathbf{N}_{2}$. For nonconforming discretizations, however, a more sophisticated algorithm is required, since these integrals involve different shape functions $\mathbf{N}_{1}$ and $\mathbf{N}_{2}$, associated with nonmatching meshes, which have to be integrated over different elements.

As detailed in Refs. [25,27], the general procedure is based on the determination of the intersection between the elements of the different meshes. For arbitrary elements in a general three-dimensional problem, this task is expected to be quite complex [22,25]. In this case, the interfaces $\Gamma_{1 c}$ and $\Gamma_{2 c}$ connected by the transfer matrix can be arbitrary curved dissimilar surfaces. The calculation of the intersection between elements can be carried out through the projection of the interfaces over an intermediate surface [22,25]. In some three-dimensional cases of practical interest, however, the coupling interfaces of the connecting subdomains are simpler. For example, exhaust devices such as oval catalytic converters [3] belong to this category. Usually, the inlet and outlet sections of the catalyst are planar and parallel, thus simplifying the problem of finding the intersection between elements in comparison with the case of general surfaces. Additional simplifications can be achieved for two-dimensional and axisymmetric configurations. The latter case will be considered in the current investigation to assess the convergence of the finite element method when nonconforming meshes and 
transfer matrices are used simultaneously. The particular test problems are depicted in Figs. 3 and 6, and described in detailed in Sec. 3, where circular catalytic converters and perforated dissipative mufflers are analysed. In such axisymmetric geometries with planar and parallel interfaces $\Gamma_{1 c}$ and $\Gamma_{2 c}$, the intersections between elements are straight lines, associated with the four possibilities depicted in Figs. 2(a)-(d) [25,27]. Details for curvilinear interfaces and more general three-dimensional problems can be found in the Refs. [22,25,27].

The algorithm for evaluating the coupling matrices $\mathbf{C}_{12}$ and $\mathbf{C}_{21}$ requires suitable loops along the interfaces $\Gamma_{1 c}$ and $\Gamma_{2 c}$ connected by the transfer matrix T. Fig. 2 shows a partial view of the subdomains $\Omega_{1}$ and $\Omega_{2}$, where the three nodes belonging to one side of a particular quadratic element are depicted over the corresponding interface. According to the figure, the finite elements located along $\Gamma_{1 c}$ and $\Gamma_{2 c}$ do not match, the associated shape functions $\mathbf{N}_{1}$ and $\mathbf{N}_{2}$ are different and hence the integrals (13) and (19) have to be taken with respect to different meshes. To proceed, it is necessary to compute the domain where the elements of $\Gamma_{1 c}$ and $\Gamma_{2 c}$ intersect. Intersection checks are carried out according to Fig. 2, where the four possibilities are shown (see grey line). Once all the intersections are defined, the integrals are calculated without overlapping or voids. The algorithm for the assembly of the coupling matrices finishes by locating the results into the right entries.

\section{RESULTS AND DISCUSSION}

\subsection{Catalytic converter}

The first numerical analysis is associated with a catalytic converter. Fig. 3 shows a scheme of the geometry associated with the axisymmetric configuration considered in the FE computations. 
According to Sec. 2, the central capillary region is replaced by a plane wave transfer matrix. In the absence of flow, the matrix considered for the monolith is given by $[2,12,13]$

$$
\mathbf{T}=\left(\begin{array}{ll}
T_{11} & T_{12} \\
T_{21} & T_{22}
\end{array}\right)=\left(\begin{array}{cc}
\cos \left(k_{m} L_{m}\right) & \frac{\mathrm{j} \rho_{m} c_{m} \sin \left(k_{m} L_{m}\right)}{\phi} \\
\frac{\mathrm{j} \phi \sin \left(k_{m} L_{m}\right)}{\rho_{m} c_{m}} & \cos \left(k_{m} L_{m}\right)
\end{array}\right) .
$$

Here, the monolith porosity is $\phi$, the length of the capillary ducts is denoted by $L_{m}, k_{m}=\omega / c_{m}$ is the wavenumber and $\rho_{m}$ and $c_{m}$ are the effective density and speed of sound $[2,12,13]$, given by

$$
\begin{gathered}
\rho_{m}=\rho_{0}\left(1+\frac{R \phi}{\mathrm{j} \omega \rho_{0}} G_{c}(s)\right), \\
c_{m}=\frac{c_{0}}{\sqrt{\left(1+\frac{R \phi}{\mathrm{j} \omega \rho_{0}} G_{c}(s)\right)(\gamma-(\gamma-1) F)}} .
\end{gathered}
$$

In Eqs. (24) and (25), $\rho_{0}$ and $c_{0}$ are the air density and speed of sound in the air (the values $\rho_{0}$ $=1.225 \mathrm{~kg} / \mathrm{m}^{3}$ and $c_{0}=340 \mathrm{~m} / \mathrm{s}$ for a temperature of $15^{\circ} \mathrm{C}$ are considered hereafter), $R$ is the steady flow resistivity, $\gamma$ is the ratio of specific heats, $s$ is the shear wave number calculated as

$$
s=\alpha \sqrt{\frac{8 \omega \rho_{0}}{R \phi}},
$$

and $F$ is given by

$$
F=\frac{1}{1+\frac{R \phi}{\mathrm{j} \operatorname{Pr} \omega \rho_{0}} G_{c}(\sqrt{\operatorname{Pr}} s)},
$$

Pr being the Prandtl number [2]. In the previous Eqs. (24), (25) and (27), $G_{c}(s)$ is given by 


$$
G_{c}(s)=\frac{-\frac{s}{4} \sqrt{-\mathrm{j}} \frac{\mathrm{J}_{1}(s \sqrt{-\mathrm{j}})}{\mathrm{J}_{0}(s \sqrt{-\mathrm{j}})}}{1-\frac{2}{s \sqrt{-\mathrm{j}}} \frac{\mathrm{J}_{1}(s \sqrt{-\mathrm{j}})}{\mathrm{J}_{0}(s \sqrt{-\mathrm{j}})}}
$$

where $\mathrm{J}_{0}$ and $\mathrm{J}_{1}$ are Bessel functions of the first kind and zeroth and first order, respectively. Finally, in Eq. (26), $\alpha$ depends on the geometry of the capillary cross-section. Eqs. (24)-(28) are valid for a monolith with identical parallel capillaries normal to the surface. Further details can be found in Ref. [13].

The following values define the selected geometry: $L_{A}=L_{E}=0.1 \mathrm{~m}, L_{B}=L_{D}=0.03 \mathrm{~m}$, $L_{m}=0.135 \mathrm{~m}, R_{A}=R_{E}=0.0268 \mathrm{~m}$ and $R_{C}=0.0886 \mathrm{~m}$. This monolith is characterised with the following properties: $R=500 \mathrm{rayl} / \mathrm{m}, \phi=0.8$ and $\operatorname{Pr}=0.7323$. For square capillary ducts, the value $\alpha=1.07$ is assumed in the calculation of the shear wave number [13].

Two different groups of nonconforming finite element discretizations are considered. The meshes of the former, denoted as Case I, have coarser meshes in the inlet region, while more refined grids are used in the outlet cavity. Case II is associated with the opposite configuration, where a more refined mesh is considered in the inlet. In this numerical example the geometry of the catalytic converter is symmetric and the discretizations of Case II are obtained by interchanging the inlet/outlet meshes of Case I. To illustrate the main features of the finite element meshes, some of the discretizations considered in this work are shown in Fig. 4. In all the cases, 8-node quadratic quadrilateral elements have been used for mesh generation. Additional relevant data (number of nodes and elements) are also detailed in the figure. As can be seen, the meshes depicted in Fig. 4(a) are nonconforming, with different discretizations along both sides of the monolith inlet/outlet faces (that has been replaced by 
the transfer matrix T). Conforming meshes are shown in Fig. 4(b), with identical grids along both sides. The nonconforming meshes depicted in the figure correspond to Case I. As indicated previously, Case II can be easily obtained by interchanging the inlet/outlet discretizations.

First, a comparison between relative errors is presented to examine the accuracy and convergence performance of the calculation algorithm for nonconforming meshes coupled with transfer matrices. The magnitudes chosen for the analysis are the four poles [1] of the catalytic converter. These are calculated according to

$$
A=\left.\frac{P_{1}}{P_{2}}\right|_{U_{2}=0}, \quad B=\left.\frac{P_{1}}{U_{2}}\right|_{P_{2}=0}, \quad C=\left.\frac{U_{1}}{P_{2}}\right|_{U_{2}=0}, \quad D=\left.\frac{U_{1}}{U_{2}}\right|_{P_{2}=0},
$$

where the subscripts 1 and 2 denote the inlet and outlet central nodes. The inlet and outlet lengths $L_{A}$ and $L_{E}$ are long enough to guarantee the decay of evanescent waves generated at the geometrical transitions. Therefore only plane waves exist at the inlet/outlet sections for the maximum frequency of the analysis [1]. The following definitions of the relative error are considered for pole $A$

$$
\begin{aligned}
\text { Error }_{\text {conf }} & =\sqrt{\frac{\sum_{i=1}^{\text {nfreq }}\left(A_{i}^{\text {conf }}-A_{i}^{\text {ref }}\right)\left(A_{i}^{\text {conf }}-A_{i}^{\text {ref }}\right)^{*}}{\sum_{i=1}^{\text {nfreq }} A_{i}^{\text {ref }} A_{i}^{\text {ref* }}},} \\
\text { Error }_{\text {nonconf }} & =\sqrt{\frac{\sum_{i=1}^{\text {nfreq }}\left(A_{i}^{\text {nonconf }}-A_{i}^{\text {ref }}\right)\left(A_{i}^{\text {nonconf }}-A_{i}^{\text {ref }}\right)^{*}}{\sum_{i=1}^{n f r e q} A_{i}^{\text {ref }} A_{i}^{\text {ref* }}}} .
\end{aligned}
$$

Similar calculations have been carried out for poles $B, C$ and $D$. In the previous Eqs. (33) and (34), nfrec is the number of frequencies included in the calculations, the asterisk denotes the complex conjugate, superscripts conf and nonconf are associated with conforming 
and nonconforming finite element computations and superscript ref is related to the reference solution. The later has been obtained with a conforming refined FE mesh consisting of 8-node quadratic quadrilateral axisymmetric elements. To guarantee an accurate reference, the discretization of this reference grid contains 16682 nodes and 5400 elements, whose size varies from a minimum value of $0.001 \mathrm{~m}$ to a maximum element edge length of $0.003 \mathrm{~m}$. This provides between 35 and 100 quadratic elements per wavelength for the maximum frequency $f_{\max }=3200 \mathrm{~Hz}$ considered in the simulations. All the calculations have been executed with frequency increments of $10 \mathrm{~Hz}$ in the range from $f_{\min }=10 \mathrm{~Hz}$ to $f_{\max }=3200 \mathrm{~Hz}$, and therefore the number of frequencies is given by $n f r e c=320$ in the summations, Eqs. (33) and (34). In Fig. 5, the relative errors are plotted against the number of nodes (in log-log scale).

As can be seen in Fig. 5, a nearly linear reduction of the error is achieved (in log-log plot) as the number of nodes is increased, for the conforming and nonconforming approaches (in this latter case for both Cases I and II). A comparison between the error curves indicates that the accuracy of the solutions associated with nonconforming meshes is slightly lower than the conforming method, at least for this particular numerical example. This is valid for all the poles and both Cases I and II. The convergence rate, however, is nearly the same in the example provided, with a slope slightly lower than unity (in absolute value). Regarding the four poles, and for the error definitions of Eqs. (33) and (34), all of them exhibit similar convergence rate characteristics, while the accuracy is slightly higher for pole B. Interchanging the meshes of the inlet/outlet regions does not alter the results significantly, at least for the configuration under analysis. The most relevant differences between Cases I and II are associated with the values of poles $\mathrm{A}$ and $\mathrm{D}$, which seem to be approximately interchanged. The performance of the nonconforming approach is valid from a practical point of view, since the relative errors achieved with the more refined meshes (3074 nodes) are 
lower than $1 \%$. The particular values shown in Fig. 5 have been computed with 8-node quadratic quadrilateral elements. As in other finite element problems dealing with nonconforming meshes [27], it is expected that the accuracy and convergence rate will progressively improve as the element shape functions increase in degree.

Table 1 shows a comparison between the computation time associated with the conforming and nonconforming approaches. In particular, node searching algorithm and mesh creation are considered. The values generated have been computed on a Core 2 Quad, 2.83GHz machine with $3 \mathrm{~GB}$ of RAM. The subroutines for node searching are implemented in Matlab, ten calculations have been running and the average value has been taken. The time for node searching is divided into two parts: location of nodes at the coupling interfaces and, after this, determination of intersections between elements of different subdomains. As can be seen, the values are very small and there are no significant differences between the computations for the geometries considered.

Regarding the mesh generation, the in-house code implemented in Matlab imports the finite element meshes created with the commercial finite element program Ansys. Mesh creation times are very small and there are no remarkable differences between matching and nonmatching grids since the geometries under analysis can be meshed with the same technique. This consists of combining quadrilateral areas (two rectangles and two trapeziums) where the element size is defined by specifying the number of divisions (number of elements) associated with each external line. This simple procedure is used to get the necessary nodal coincidence required by the conforming approach. Its application is possible due to the simplicity of the geometries under consideration. In the case of problems requiring arbitrary three-dimensional meshes, the achievement of conforming meshes is not always simple. 


\subsection{Perforated dissipative muffler}

The second example considered in the current investigation is related to a perforated dissipative muffler. The relevant features of the geometry under analysis are depicted in Fig. 6. This configuration is chosen to analyse a problem where the coupling interfaces are parallel to the main axial direction (from an acoustical point of view). This is in contrast with the previous catalyst problem, where the connecting boundaries were normal to the main direction of propagation. Both sides of the perforated screen are coupled by the transfer matrix $\mathbf{T}$, which contains the acoustic impedance. For the sake of clarity, these sides are represented as separated dashed lines in Fig. 6, although two overlapped lines are used in the finite element meshes. The main geometrical dimensions of the selected configuration are: $L_{A}$ $=L_{C}=0.1 \mathrm{~m}, L_{B}=0.2 \mathrm{~m}, R_{1}=0.0268 \mathrm{~m}$ and $R_{2}=0.0886 \mathrm{~m}$.

The outer chamber between radii $R_{1}$ and $R_{2}$ is filled with a homogeneous and isotropic absorbent material, characterized by the following complex values of characteristic impedance $\tilde{Z}=\tilde{\rho} \tilde{c}$ and wavenumber $\tilde{k}=\omega / \tilde{c}$ [17]

$$
\begin{gathered}
\tilde{Z}=Z_{0}\left(\left(1+0.09534\left(\frac{f \rho_{0}}{R}\right)^{-0.754}\right)+\mathrm{j}\left(-0.08504\left(\frac{f \rho_{0}}{R}\right)^{-0.732}\right)\right), \\
\tilde{k}=k_{0}\left(\left(1+0.16\left(\frac{f \rho_{0}}{R}\right)^{-0.577}\right)+\mathrm{j}\left(-0.18897\left(\frac{f \rho_{0}}{R}\right)^{-0.595}\right)\right) .
\end{gathered}
$$

Here, $Z_{0}=\rho_{0} c_{0}$ is the characteristic impedance of air, $k_{0}=\omega / c_{0}$ is the wavenumber, $\tilde{\rho}$ and $\tilde{c}$ are the equivalent density and speed of sound for the absorbent material [13], respectively, $f$ is the frequency, and $R$, as in the previous case of the monolith, the steady flow resistivity, 
given by $4896 \mathrm{rayl} / \mathrm{m}$ for a bulk density of $100 \mathrm{~kg} / \mathrm{m}^{3}$ (see Ref. [17] for further details). This absorbent material is confined by a concentric perforated screen whose acoustic impedance is denoted by $\tilde{Z}_{p}$. In the FE simulations, the perforated surface is replaced by a transfer matrix given by

$$
\mathbf{T}=\left(\begin{array}{ll}
T_{11} & T_{12} \\
T_{21} & T_{22}
\end{array}\right)=\left(\begin{array}{cc}
1 & \tilde{Z}_{p} \\
0 & 1
\end{array}\right)
$$

The acoustic impedance is written as $[1,20]$

$$
\tilde{Z}_{p}=Z_{0} \frac{\left(0.006+\mathrm{j} k_{0}\left(t_{p}+0.425 d_{h}\left(1+\frac{\tilde{\rho}}{\rho_{0}}\right) F(\sigma)\right)\right)}{\phi}
$$

$\phi$ being the porosity, $t_{p}$ the thickness and $d_{h}$ the hole diameter. The expression detailed in Eq. (38) includes the influence of the absorbent material (by means of $\tilde{\rho}$ ) on the behaviour of the perforations, as well as the acoustic interaction between holes, defined by the function $F(\phi)$. The average value of Ingard's and Fok's corrections is used [20]

$$
F(\phi)=1-1.055 \sqrt{\phi}+0.17(\sqrt{\phi})^{3}+0.035(\sqrt{\phi})^{5}
$$

In all the computations hereafter, the numerical values associated with the perforated surface are $\phi=0.1(10 \%), t_{p}=0.001 \mathrm{~m}$ and $d_{h}=0.0035 \mathrm{~m}$.

Two nonconforming groups are distinguished, as in Section 3.1. The meshes of the former, Case I, have coarser discretizations in the dissipative region in comparison with the central perforated pipe. For Case II, the opposite situation is considered. Some of the finite element meshes considered in the computations are shown in Fig. 7. All the discretizations have been generated with 8-node quadratic quadrilateral elements. Fig. 7 also provides basic information such as the number of nodes and elements. Different discretizations along both 
sides of the perforated pipe are depicted in Figs. 7(a) and 7(b) for Cases I and II, respectively, while the conforming grids are sketched in Fig. 7(c).

To assess the algorithm performance in terms of accuracy and convergence, the finite element results are analysed as follows. The four poles, calculated from the acoustic pressure and axial velocity at the central inlet/outlet nodes, are considered again. The expressions for the computation of the relative error are given by Eqs. (33) and (34). Here, in order to be confident of an accurate reference solution, an analytical mode matching calculation has been obtained including 20 axisymmetric modes [17,20]. As in Sec. 3.1, all the computational tests have been calculated with frequency increments of $10 \mathrm{~Hz}$ ranging from $f_{\min }=10 \mathrm{~Hz}$ to $f_{\max }=$ $3200 \mathrm{~Hz}$. The relative errors associated with the muffler four poles are depicted in Fig. 8 in $\log -\log$ scale.

In all the cases the error curves are approximately linear, at least for increasing number of nodes. Initially, the conforming approach exhibits the best performance in terms of accuracy and convergence rate. This behaviour is no longer kept as the number of nodes increases. As can be seen in Fig. 8, the nonconforming meshes associated with Case I (coarser discretization in the outer dissipative region) perform well when compared to the conforming ones. This situation has been also observed in the literature devoted to acoustic problems for a spherical pulse [25], where nonconforming solutions can beat conforming predictions in some cases. Nevertheless, the nonconforming results related to Case II (finer discretization in the outer chamber) do not improve at the same rate as Case I. The accuracy of the Case II solution is lower than the conforming one in all the cases and the convergence rate is nearly the same. One of the possible reasons for this behaviour of the nonconforming approach (Case II) in the particular problem under consideration may be related to over discretization of the outer 
dissipative chamber. This region is likely to have less influence in the main direction of propagation. Concerning the four poles, the general trend is similar for all four parameters, with pole B exhibiting a slightly higher accuracy (as in the case of the catalytic converter, Sec. 3.1). To conclude, the nonconforming approach performs well for both types of meshes (Cases I and II) since relative errors lower than $0.1 \%$ are obtained for the more refined finite element meshes (1226 nodes for Case I and 2090 nodes for Case II).

\section{CONCLUSIONS}

A finite element algorithm that combines transfer matrices and nonconforming meshes has been implemented to analyse the acoustic behaviour of exhaust devices consisting of several subdomains. The use of nonmatching grids at the connecting interfaces between subdomains increases the flexibility of the procedure and simplifies the mesh generation process. The technique allows to handle arbitrary meshes where the nodes do not coincide at the coupling boundaries. Therefore the grid information associated with a particular region is independent of the remaining subdomains.

Two numerical examples are presented to illustrate the validity and convergence performance of the proposed technique. In the first case, the connecting interfaces are normal to the main direction of propagation. The particular configuration consists of a catalytic converter in which the monolith is replaced by a transfer matrix. Therefore, only plane wave propagation is assumed in the capillary ducts. Finite element discretizations are used to compute the multidimensional acoustic fields in the rest of catalyst subcomponents (inlet/outlet and tapered ducts), where three-dimensional waves can exist. Two kinds of nonconforming meshes are considered, depending on the side (inlet or outlet) having a more 
refined discretization, whose results do not differ significantly. The comparison with conforming predictions shows that the accuracy of the solutions associated with nonconforming meshes is slightly lower, while the convergence rate is nearly the same. From a practical point of view, the nonconforming approach provides suitable results, with relative errors lower than $1 \%$ for the more refined meshes of the particular catalytic converter under analysis.

The second example is a perforated dissipative muffler, where the coupling interfaces are parallel to the main direction of propagation. Concerning the finite element modelling, the perforated duct can be replaced by a transfer matrix where the off-diagonal term $(1,2)$ equals its acoustic impedance $\tilde{Z}_{p}$. Nonconforming meshes are considered with finer elements in the duct and a coarser mesh in the outer chamber (Case I), and vice versa (Case II). In contrast with the catalyst problem, significant discrepancies are found between Case I and Case II. Although the conforming predictions present the best performance for coarse discretizations, the nonconforming technique performs very well when the number of nodes increases (Case I grids). It is shown here that the nonconforming method is capable of computations with accuracy and convergence rate comparable to the conforming approach. For very refined meshes, these nonconforming computations are even better than the conforming predictions. The performance of the nonconforming meshes related to Case II is not as good as Case I. Anyway, the behaviour of the nonconforming approach for both types of meshes seems suitable since relative errors lower than $0.1 \%$ can be achieved with the more refined finite element grids.

Some aspects of the nonconforming approach presented in the current paper are relevant for future investigations, which might be related to the presence of mean flow, the 
improvement of the accuracy and the application to additional devices of the breathing system of internal combustion engines, such as diesel particulate filters.

\section{ACKNOWLEDGMENT}

Authors gratefully acknowledge the financial support of Ministerio de Ciencia e Innovación and the European Regional Development Fund by means of the projects DPI2007-62635 and DPI2010-15412. 


\section{REFERENCES}

[1] Munjal ML. Acoustics of Ducts and Mufflers. New York: Wiley-Interscience; 1987.

[2] Selamet A, Easwaran V, Novak JM, Kach RA. Wave attenuation in catalytic converters: Reactive versus dissipative effects. J Acoust Soc Am 1998; 103: 935-43.

[3] Selamet A, Kothamasu V, Novak JM, Kach RA. Experimental investigation of in-duct insertion loss of catalysts in internal combustion engines. Appl Acoust 2000; 60: 451-87.

[4] Allam S, Åbom M. Modeling and testing of after-treatment devices. J Vib Acoust 2006; 128: 347-56.

[5] Allam S, Åbom M. Sound propagation in an array of narrow porous channels with application to diesel particulate filters. J Sound Vib 2006; 291: 882-901.

[6] Knutsson M, Åbom M. Sound propagation in narrow tubes including effects of viscothermal and turbulent damping with application to charge air coolers. J Sound Vib 2009; 320: 289-321.

[7] Peat KS, Rathi KL. A finite element analysis of the convected acoustic wave motion in dissipative silencers. J Sound Vib 1995; 184: 529-45.

[8] Wu TW. Boundary Element Acoustics. Fundamentals and Computer Codes. Southampton: WIT Press; 2000.

[9] LMS International. LMSVirtual.Lab, Rev 7B, (2007).

[10] Wu TW, Cheng CYR. Boundary element analysis of reactive mufflers and packed silencers with catalyst converters. Electr J Bound Elem 2003; 1: 218-235. 
[11] Jiang C, Wu TW, Xu MB, Cheng CYR. BEM modeling of mufflers with diesel particulate filters and catalytic converters. Noise Control Eng J 2010; 58: 243- 50.

[12] Denia FD, Antebas AG, Kirby R, Fuenmayor FJ. Multidimensional acoustic modelling of catalytic converters. The Sixteenth International Congress on Sound and Vibration 2009: Kraków, Poland, July 5-9.

[13] Allard JF. Propagation of Sound in Porous Media. London: Elsevier; 1993.

[14] Ji ZL, Selamet A. Boundary element analysis of three-pass perforated duct mufflers. Noise Control Eng J 2000; 58: 151-56.

[15] Kar T, Sharma PPR, Munjal ML. Analysis and design of composite/folded variable area perforated tube resonators for low frequency attenuation. J. Acoust Soc Am 2006; 119: 3599609.

[16] Panigrahi SN, Munjal ML. A generalized scheme for analysis of multifarious commercially used mufflers. Appl Acoust 2007; 68: 660-681.

[17] Selamet A, Xu MB, Lee IJ, Huff NT. Analytical approach for sound attenuation in perforated dissipative silencers. J Acoust Soc Am 2004; 115: 2091-99.

[18] Ji ZL. Boundary element analysis of a straight-through hybrid silencer. J Sound Vib 2006; 292: 415-23.

[19] Albelda J, Denia FD, Torres MI, Fuenmayor FJ. A transversal substructuring mode matching method applied to the acoustic analysis of dissipative mufflers. J Sound Vib 2007; 303: 614-31.

[20] Kirby R, Denia FD. Analytic mode matching for a circular dissipative silencer containing mean flow and a perforated pipe. J Acous Soc Am 2007; 122: 3471-82. 
[21] Kirby R. A comparison between analytic and numerical methods for modelling automotive dissipative silencers with mean flow. J Sound Vib 2009; 325: 565-82.

[22] Puso MA. A 3D mortar method for solid mechanics. Int J Numer Methods Eng 2004; 59: $315-36$.

[23] Zienkiewicz OC, Taylor RL, Zhu JZ. The Finite Element Method: Its Basis and Fundamentals. Oxford: Elsevier Butterworth-Heinemann; 2005.

[24] Tur M, Fuenmayor FJ, Wriggers P. A mortar-based frictional contact formulation for large deformations using Lagrange multipliers. Comput Methods Appl Mech Eng 2009; 198: 2860-73.

[25] Flemisch B, Kaltenbacher M, Wohlmuth BI. Elasto-acoustic and acoustic-acoustic coupling on non-matching grids. Int J Numer Methods Eng 2006; 67: 1791-1810.

[26] Walsh T, Reese G, Dohrman C, Rouse J. Finite element methods for structural acoustics on mismatched meshes. J Comput Acoust 2009; 17: 247-75.

[27] Triebenbacher S, Kaltenbacher M, Wohlmuth B, Flemisch B. Applications of the mortar finite element method in vibroacoustics and flow induced noise computations. Acta Acust/Acust; 96: 536-53. 


\section{FIGURE CAPTIONS}

Fig. 1. (a) Acoustic device consisting of several subdomains. (b) FE subdomains 1 and 2 connected by a transfer matrix replacing $\Omega_{c}$. Nonconforming interfaces $\Gamma_{1 c}$ and $\Gamma_{2 c}$.

Fig. 2. Intersection of two nonconforming discretizations.

Fig. 3. Geometry of the catalytic converter (monolith replaced by a transfer matrix).

Fig. 4. FE discretizations. (a) Nonconforming meshes, Case I. (b) Conforming meshes.

Fig. 5. Relative error of the finite element solutions for a catalytic converter. (a) Pole $A$. (b) Pole $B$. (c) Pole $C$. (d) Pole $D:-\mathrm{x}-$, nonconforming meshes, Case I; ———, nonconforming meshes, Case II; ——_, conforming meshes.

Fig. 6. Geometry of the perforated dissipative muffler.

Fig. 7. FE discretizations. (a) Nonconforming meshes, Case I. (b) Nonconforming meshes, Case II. (c) Conforming meshes.

Fig. 8. Relative error of the finite element solutions for a perforated dissipative muffler. (a) Pole $A$. (b) Pole B. (c) Pole C. (d) Pole $D$ : $-\mathrm{x}-$, nonconforming meshes, Case I; - - nonconforming meshes, Case II; ——_, conforming meshes. 


\section{HIGHLIGHTS}

A technique that combines transfer matrices and nonconforming FE meshes is presented A more flexible FE modelling is achieved and the mesh generation process is simplified A catalytic converter and a perforated dissipative muffler are analysed The assessment of the accuracy and convergence performance is satisfactory 


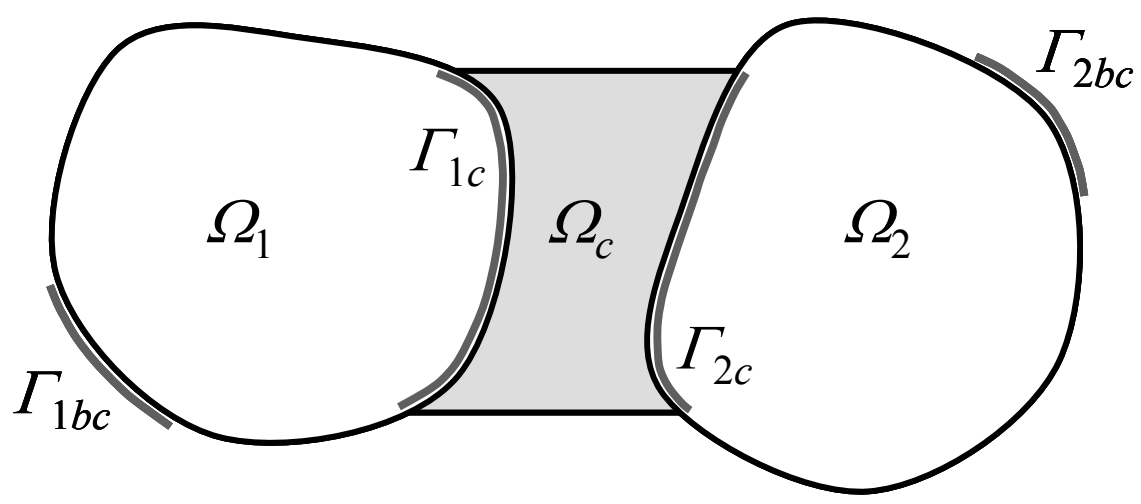

(a)

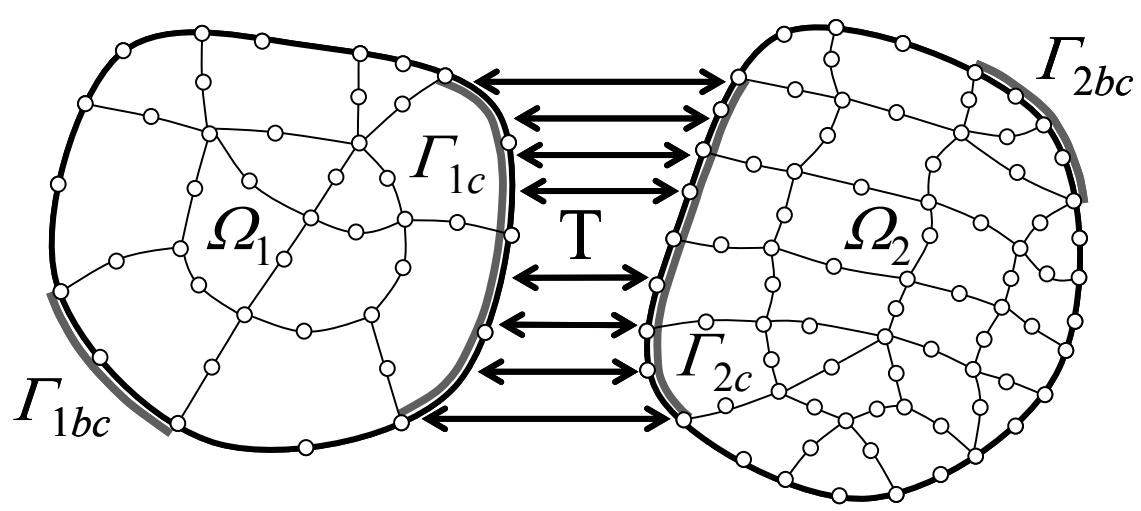

(b)

FIGURE 1 

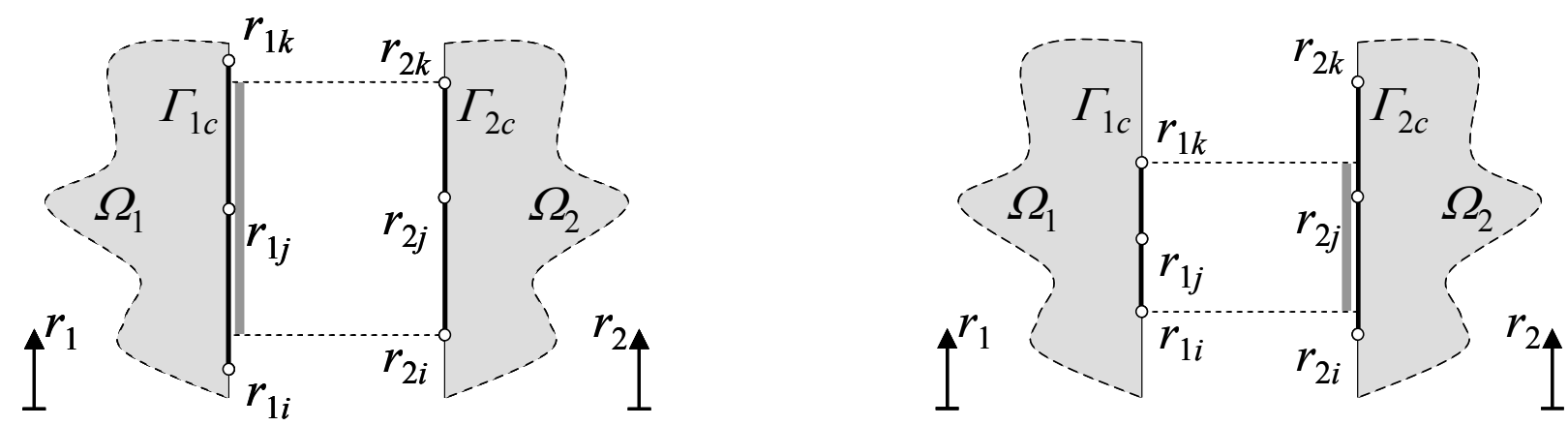

$$
r_{1 i} \leq r_{2 i}, \quad r_{2 k} \leq r_{1 k} \quad \text { (a) }
$$

$$
r_{2 i}<r_{1 i}, \quad r_{1 k}<r_{2 k}
$$
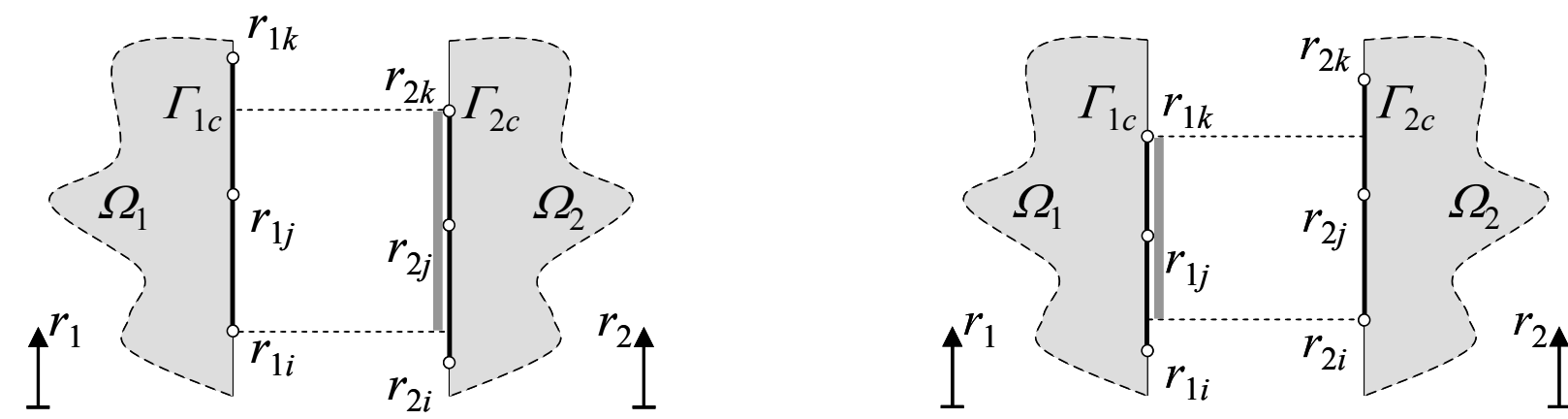

$$
r_{2 i}<r_{1 i}, \quad r_{2 k} \leq r_{1 k} \quad \text { (c) }
$$

$$
r_{1 i} \leq r_{2 i}, \quad r_{1 k}<r_{2 k}
$$

FIGURE 2 


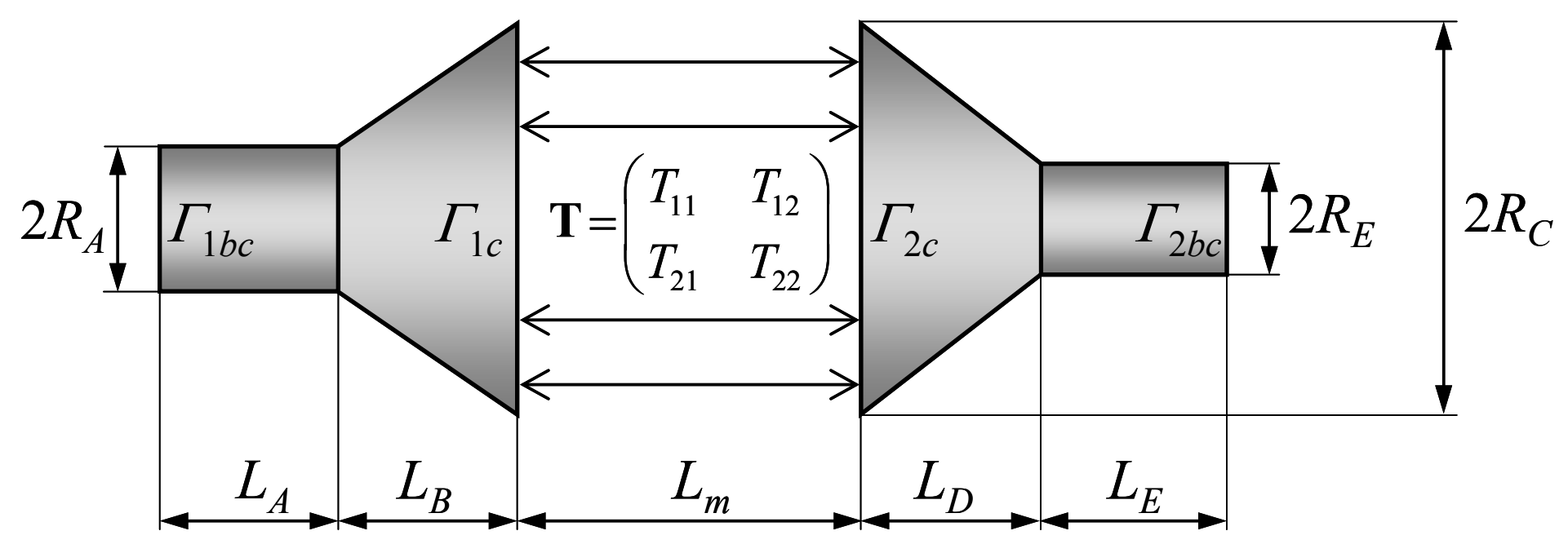

FIGURE 3 


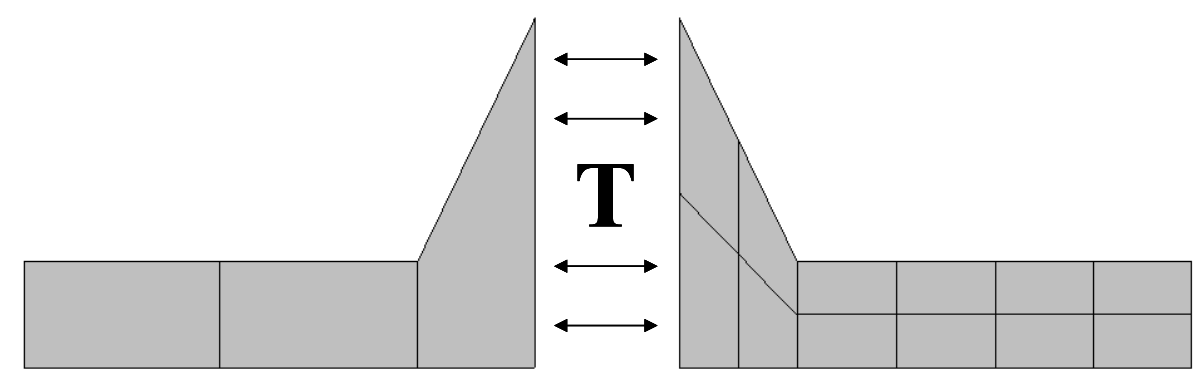

Nodes: 71. Elements: 15.

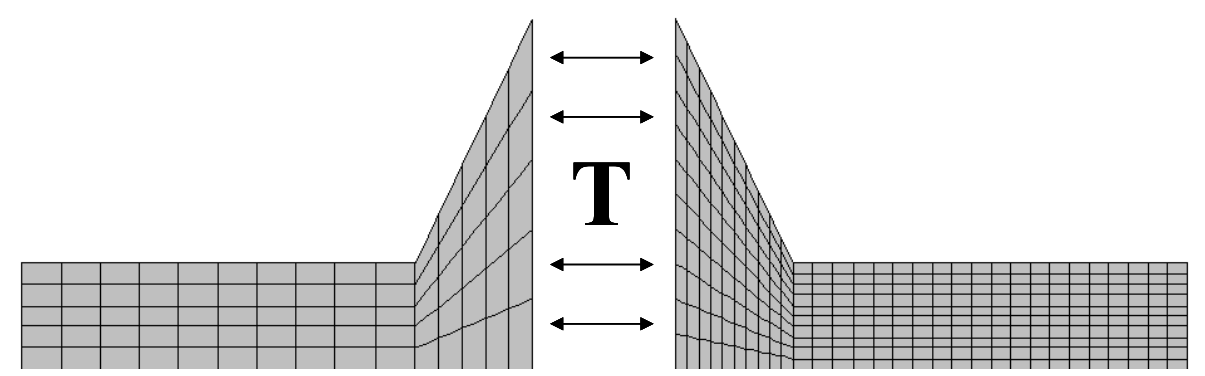

Nodes: 1247. Elements: 375.

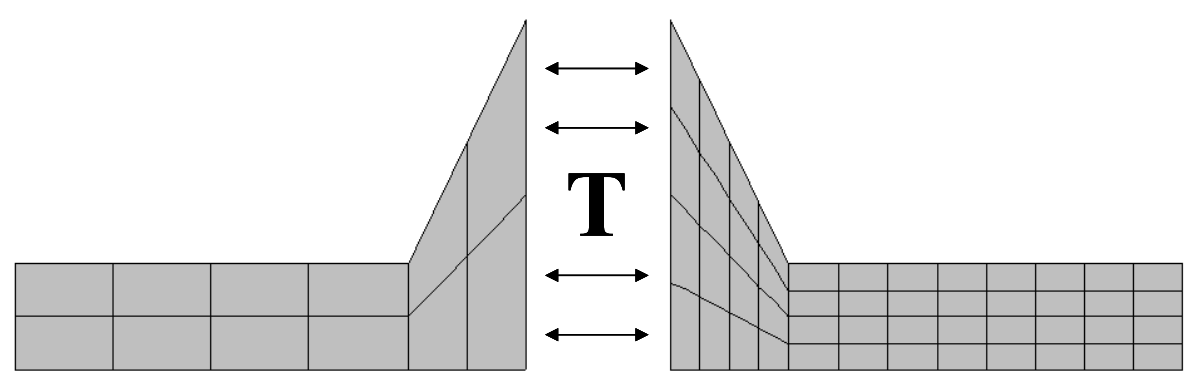

Nodes: 230. Elements: 60.

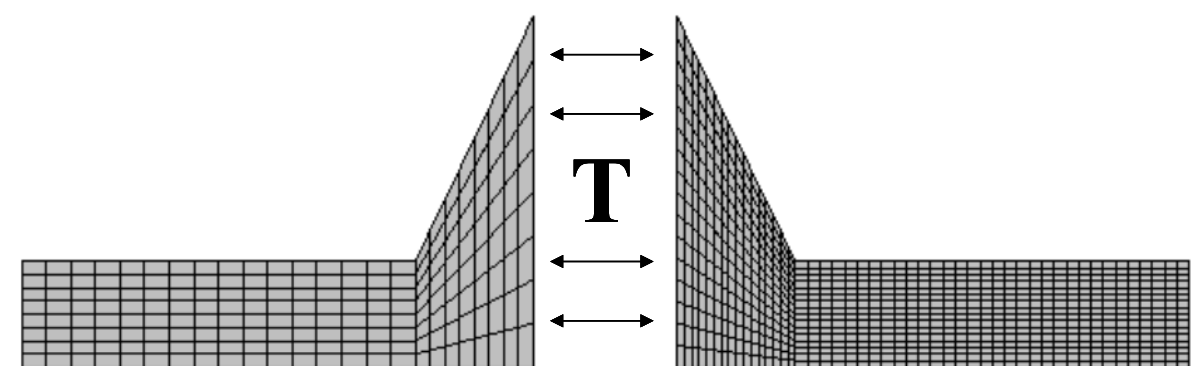

Nodes: 3074. Elements: 960. 


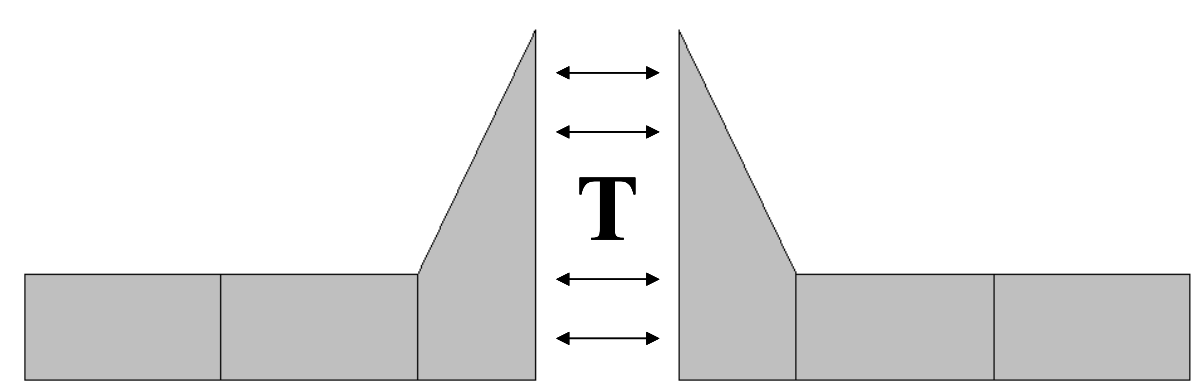

Nodes: 36. Elements: 6.

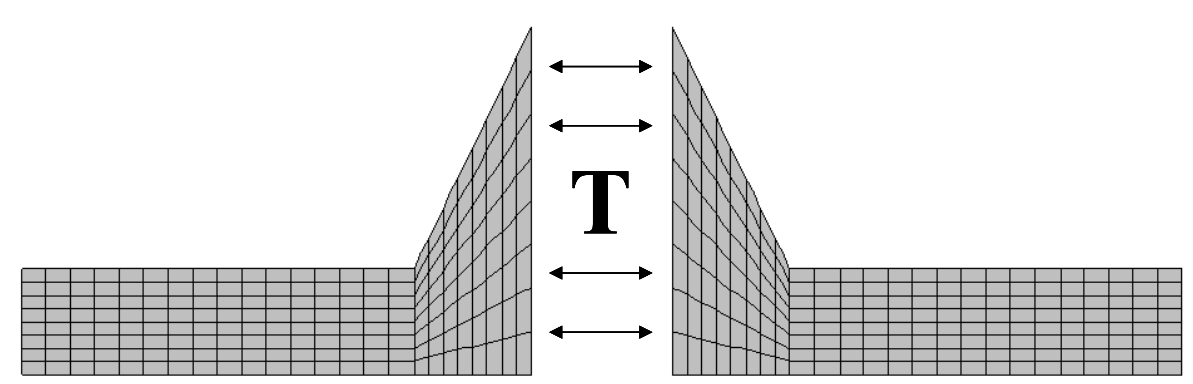

Nodes: 1282. Elements: 384.

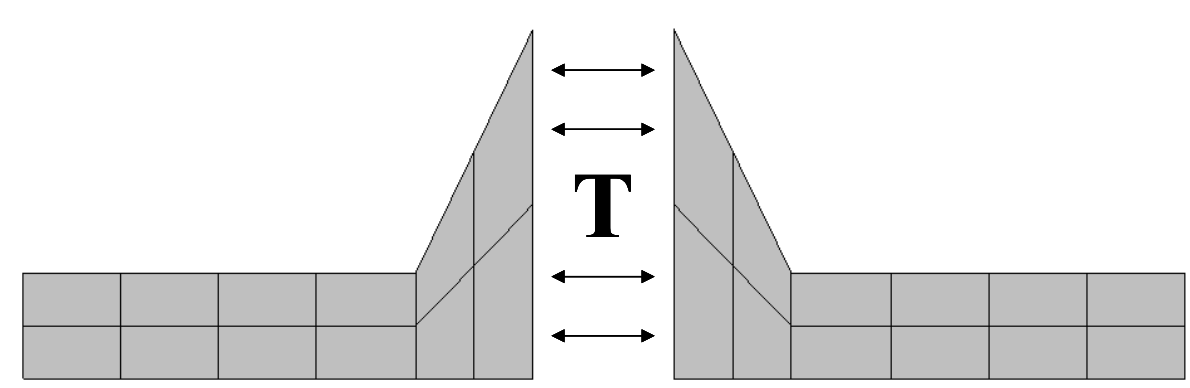

Nodes: 106. Elements: 24.

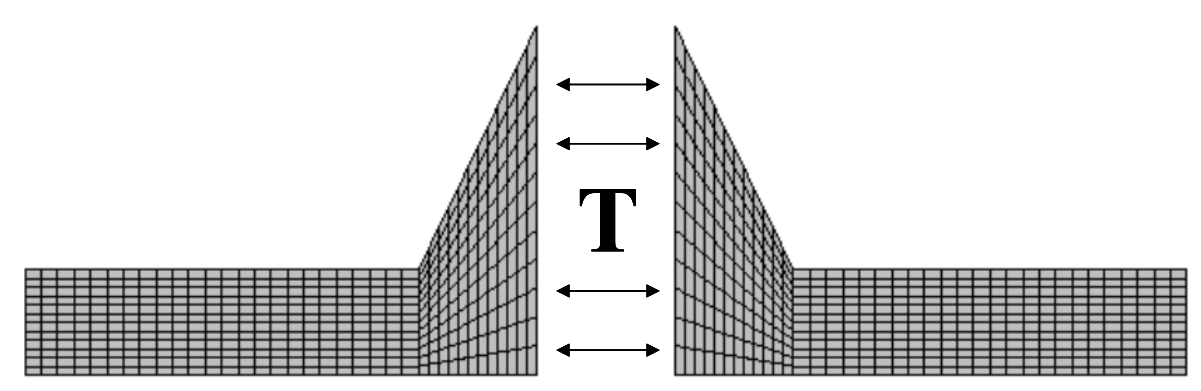

Nodes: 2786. Elements: 864. (b)

FIGURE 4(b) 

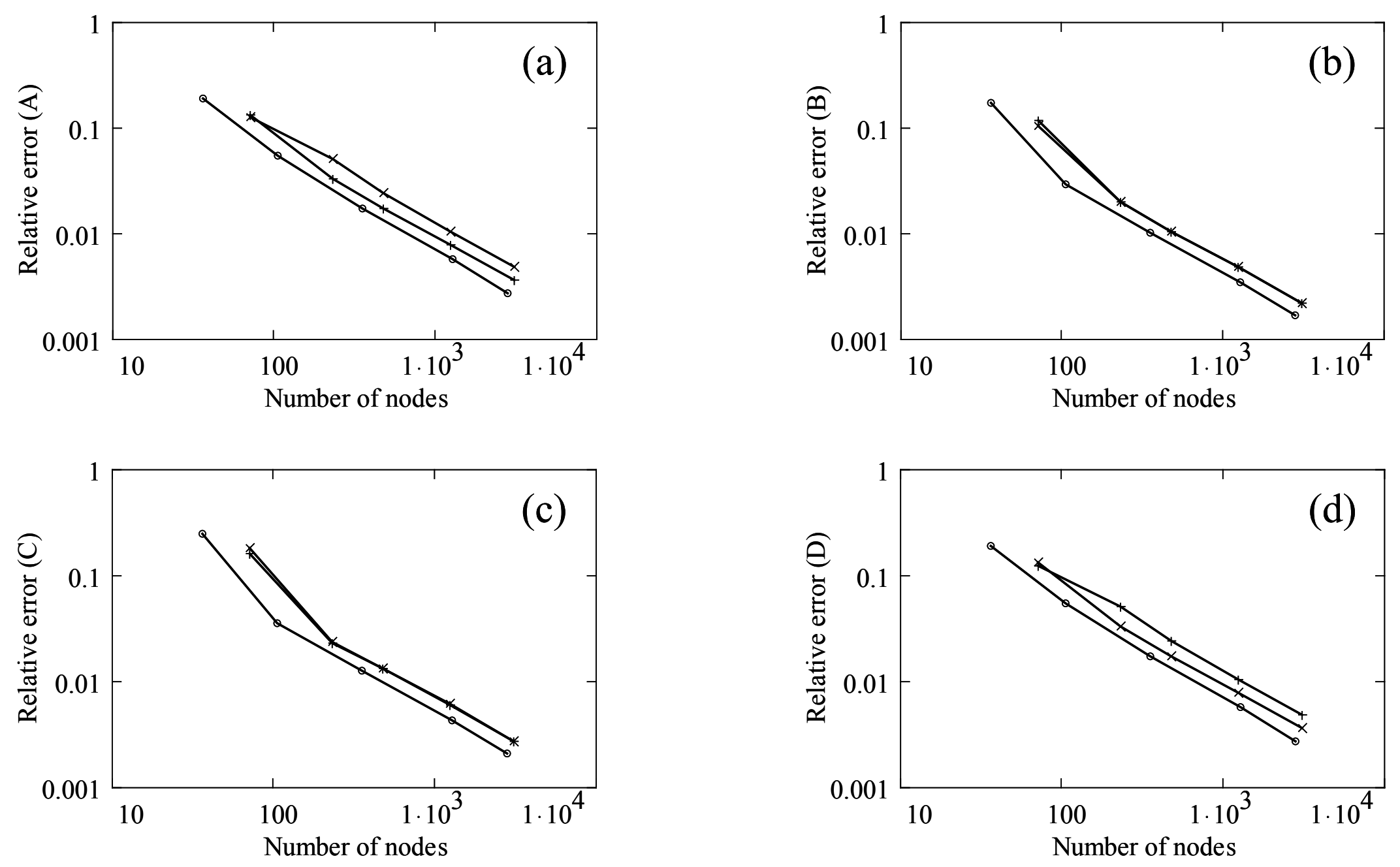

FIGURE 5 


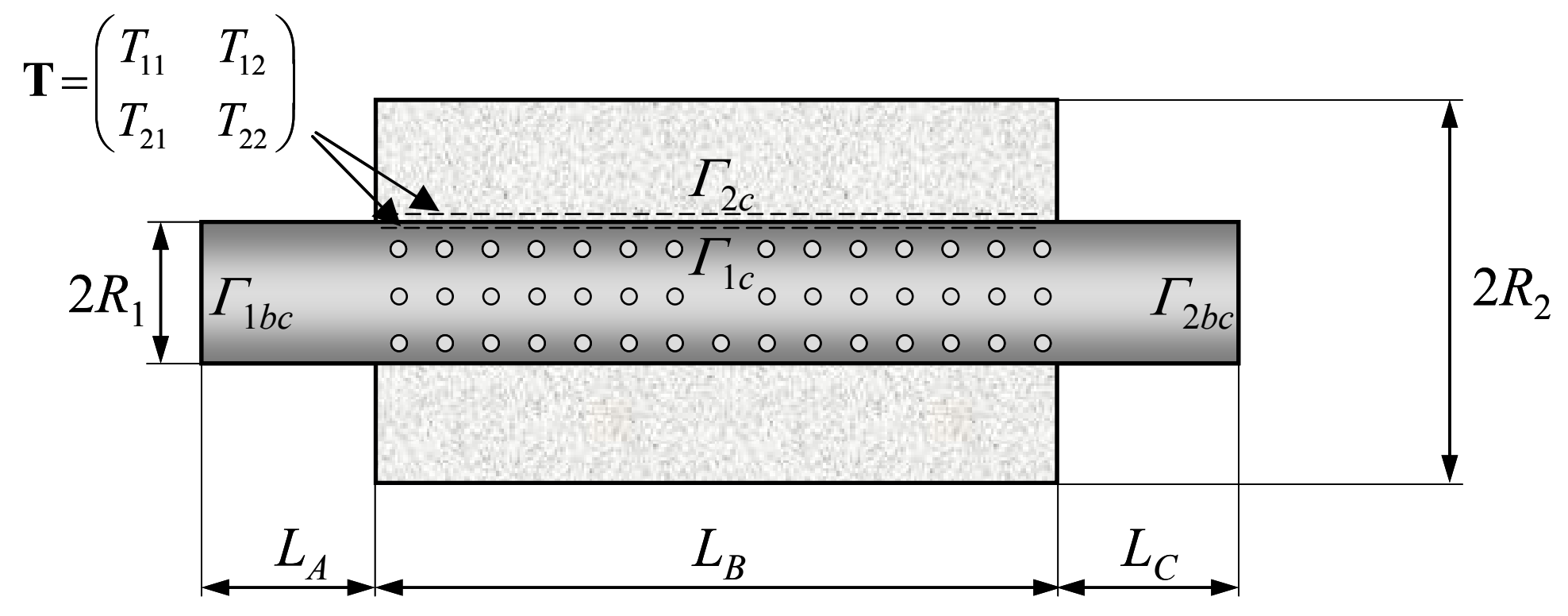

FIGURE 6 


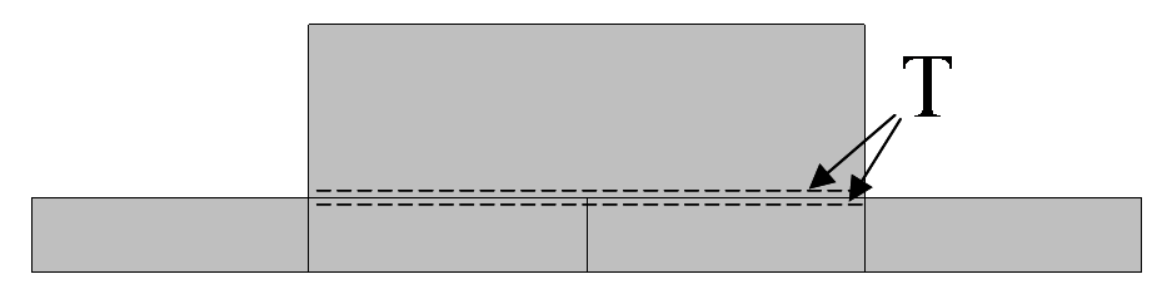

Nodes: 31. Elements: 5.

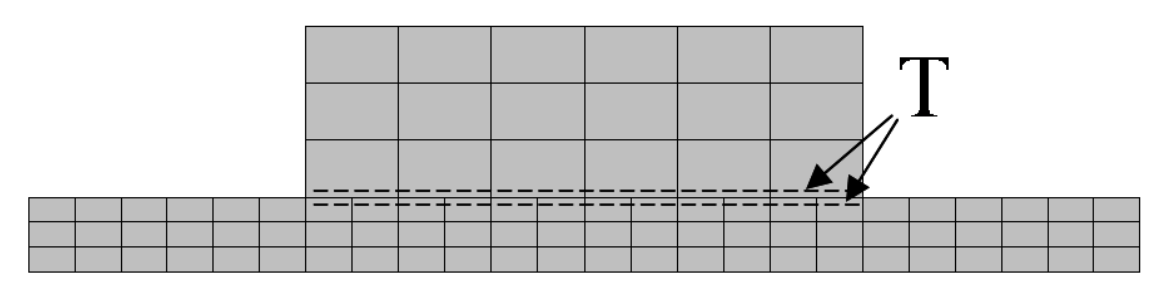

Nodes: 344. Elements: 90.

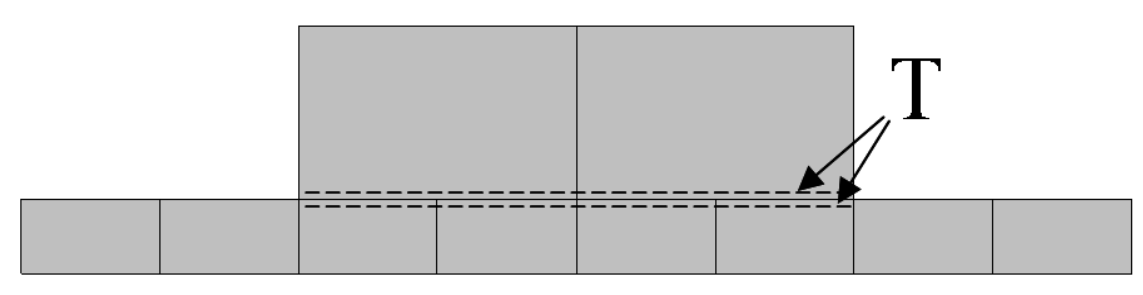

Nodes: 56. Elements: 10.

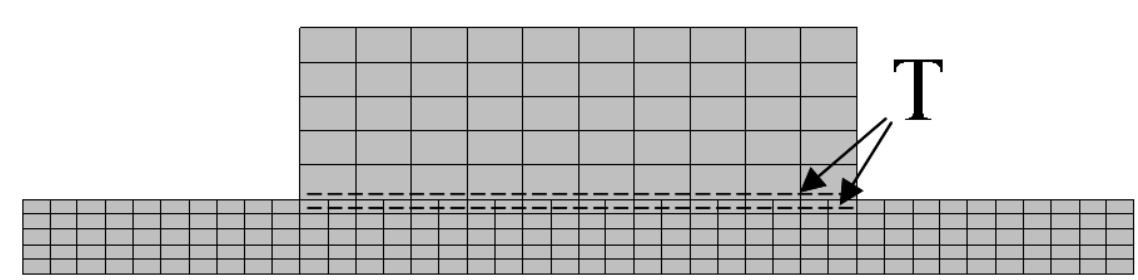

Nodes: 872. Elements: 250.

FIGURE 7(a) 


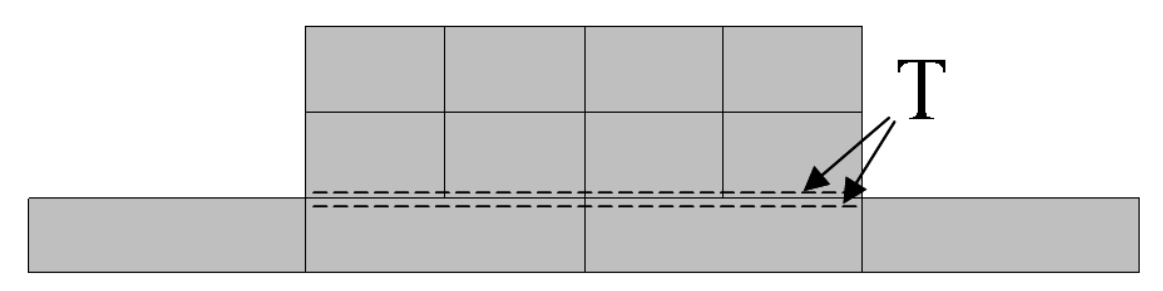

Nodes: 60. Elements: 12.

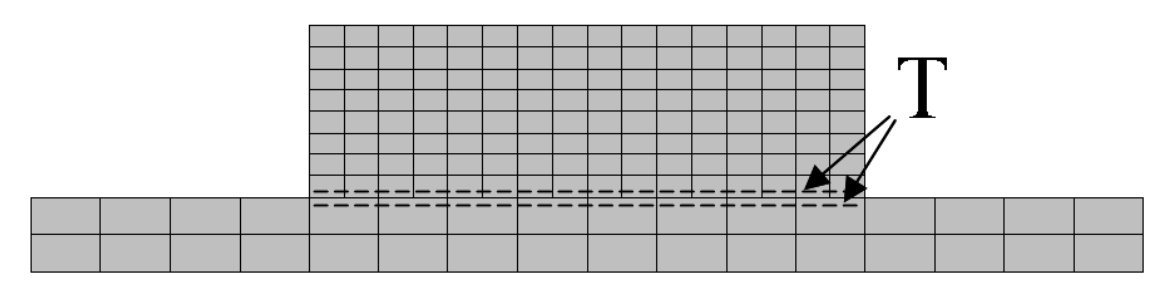

Nodes: 566. Elements: 160.

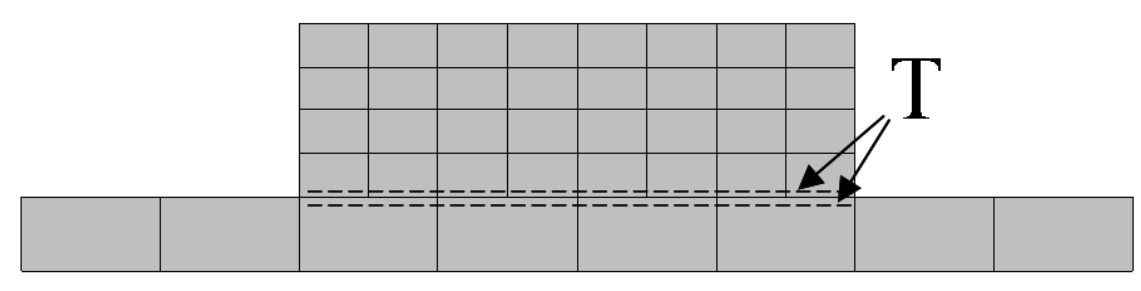

Nodes: 164. Elements: 40.

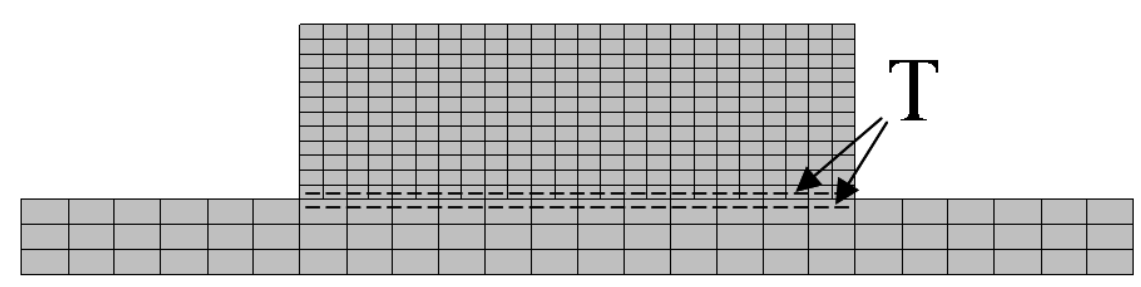

Nodes: 1208. Elements: $360 . \quad$ (b)

FIGURE 7(b) 


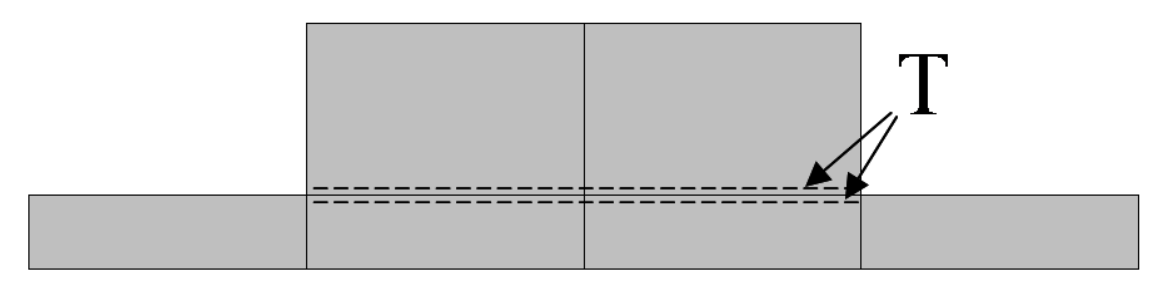

Nodes: 36. Elements: 6.

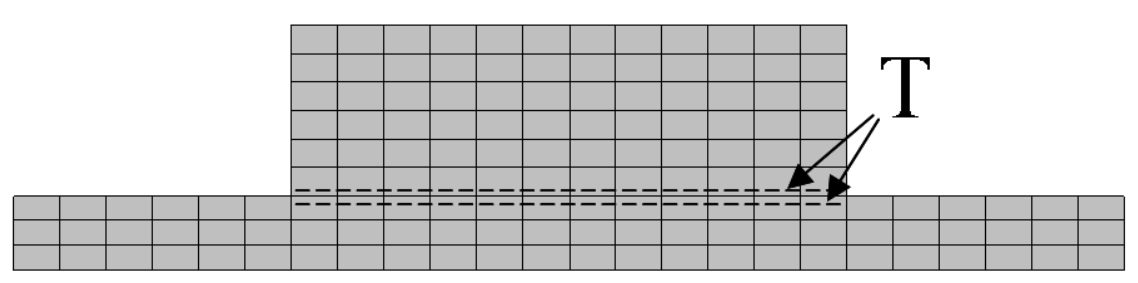

Nodes: 524. Elements: 144.

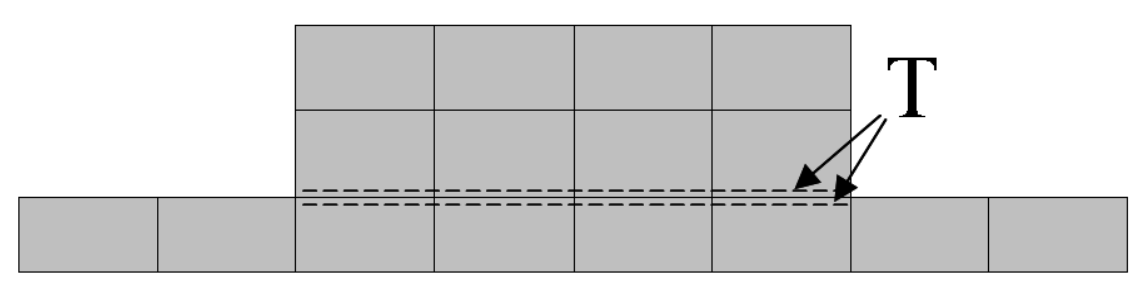

Nodes: 80. Elements: 16.

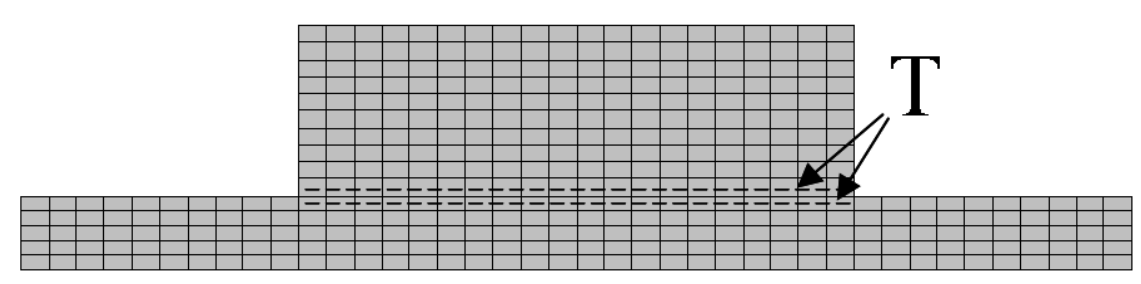

Nodes: 1352. Elements: 400.

FIGURE 7(c) 

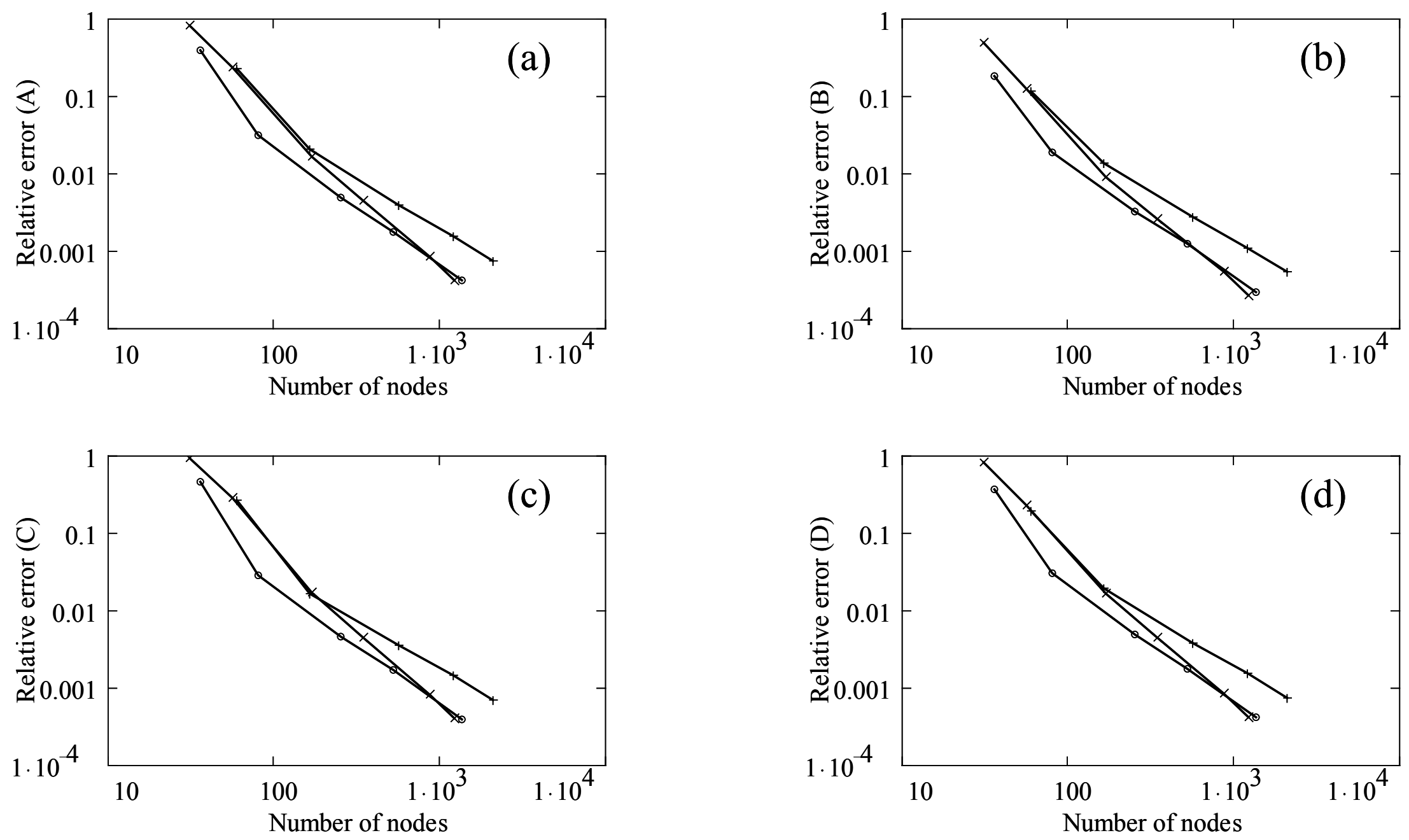

FIGURE 8 